\title{
Application of Serum Ferritin Combined with Blood Routine Testing in the Screening of Obstetrics and Gynecology Diseases
}

\author{
Yueliao Ma1", Zhiyong Qin 2, Chundong Qiü\#, Xiaohua Huang4\# \\ ${ }^{1}$ Laboratory Department of Maternal and Child Health Hospital, Hechi, China \\ ${ }^{2}$ Inspection Department of Guiping People's Hospital, Guigang, China \\ ${ }^{3}$ First People's Hospital of Yulin, Yulin, China \\ ${ }^{4}$ Department of Traditional Chinese Medicine of Yunyang County, Chongqing, China \\ Email:56835477@qq.com, qinzhiyong0866@163.com, ${ }^{\text {"101012657363@qq.com, } 54076125 @ q q . c o m}$
}

How to cite this paper: Ma, Y.L., Qin, Z.Y., Qiu, C.D. and Huang, X.H. (2021) Application of Serum Ferritin Combined with Blood Routine Testing in the Screening of Obstetrics and Gynecology Diseases. Advances in Reproductive Sciences, 9, 118-128.

https://doi.org/10.4236/arsci.2021.92012

Received: February 7, 2021

Accepted: April 23, 2021

Published: April 26, 2021

Copyright $\odot 2021$ by author(s) and Scientific Research Publishing Inc. This work is licensed under the Creative Commons Attribution International License (CC BY 4.0).

http://creativecommons.org/licenses/by/4.0/

\begin{abstract}
Objective: To understand the application of serum ferritin combined with blood routine testing in the screening of obstetrics and gynecology diseases in this region. Methods: From January 1, 2017, to October 28, 2020, all obstetric pregnant women, inpatient maternal, gynecological outpatient, and gynecological inpatients in our hospital's outpatient and inpatient clinics were collected for serum ferritin determination and blood routine related index detection and analysis Changes in results. The application value of serum special protein in gynecological and obstetrical disease screening was put forward. Results: A total of 15,656 cases of obstetrics and gynecology patients were collected, of which 15,300 were pregnant women. Comparing maternity checkups with hospitalized puerpera, the difference of all indexes except RBC was statistically significant $(P<0.05)$; Comparison of various indicators between gynecological outpatient clinics and gynecological hospitalizations, maternity checkups and gynecological outpatient clinics, inpatient maternal and gynecological hospitalizations, etc.; there was no statistically significant difference in all indicators $(\mathrm{P}>0.05)$. Comparison of hospitalized puerpera and gynecological hospitalization, the difference of other indexes except for $\mathrm{MCV}, \mathrm{MCH}$ was statistically significant $(\mathrm{P}<0.05)$; Comparing hospitalized puerpera and gynecological clinics, the difference of HGB results was statistically significant $(\mathrm{P}<0.05)$, but the difference of other indexes was not statistically significant $(P>0.05)$. Conclusion: During the maternity check-up period, the ferritin consumption is too large, the blood volume increases, and the blood routine-related items are reduced. The consumption is greater for a
\end{abstract}

${ }^{*}$ Co-first authors; ${ }^{*}$ Corresponding authors. 
period of time after delivery, and recovery requires a certain process, which is relatively low; The ferritin in the body slowly recovered and the ferritin concentration increased. This process is a process of physiological change and does not involve related diseases, but from this result, we are also required to strengthen nutrition for pregnant women in order to give birth to healthier babies. The ferritin test of gynecological patients is mainly a screening of related diseases, and the results of this time did not screen out serious diseases.

\section{Keywords}

Ferritin, Pregnant Women, Obstetrics, Gynecology, Disease Screening

\section{Introduction}

Ferritin is a soluble tissue protein that stores iron in the body. The serum of normal people contains a small amount of ferritin, but different detection methods have different normal values. Generally, the average value is about $80-130$ $\mathrm{ug} / \mathrm{L}(80-130 \mathrm{ng} / \mathrm{ml}$ for men and 35 - $55 \mathrm{ug} / \mathrm{L}$ ( $35-55 \mathrm{ng} / \mathrm{ml})$ for women [1] [2] [3] [4] [5]. The positive rate of liver cancer patients reported was as high as $90 \%$ in China. A type of iron-storing protein widely found in animals and plants. It is most abundant in the liver and spleen of mammals. Its outer diameter is about $12-14 \mathrm{~nm}$, and the cavity diameter is about $6 \mathrm{~nm}$. The outer shell (that is, apoferritin) is composed of 24 subunits, each subunit contains about 163 amino acid residues, and each molecule can bind up to 4500 Iron atoms. The molecular weight is about $450 \mathrm{kd}$. Iron-bound ferritin is "soluble" in water, and the concentration of plasma ferritin is directly proportional to the iron stored in the body [6] [7] [8] [9] [10]. There are many nutrients in the human body, such as calcium, iron, zinc, selenium, etc., which are important substances for maintaining health. There is also a kind of ferritin that is also very important to human health, especially the iron protein in pregnant women. The ferritin value in the body of women after pregnancy is relatively higher than usual, which is caused by the need for ferritin in the growth and development of the baby. Under normal conditions, the iron protein value in pregnant women should be kept between $35 \mathrm{ug} / \mathrm{L}$ and $55 \mathrm{ug} / \mathrm{L}$. As long as the actual iron protein value of pregnant women is within this range, pregnant women do not have to worry, proving that the body is not iron deficient. Maintain a normal diet [11] [12] [13] [14] [15]. The purpose of testing the ferritin content in the blood is to check whether the pregnant woman is anaemic. If the iron protein value in the pregnant woman is lower than the reference value, it proves that the iron stored in the pregnant woman is consumed, which leads to the phenomenon of iron deficiency anemia. At this time, it is necessary to take some drugs in time to supplement the iron element to avoid affecting the health of the fetus. For pregnant women with low levels of ferritin in their bodies, iron supplementation is an urgent matter; The pregnant women not only take some iron supplements but also need dietary 
treatment. When pregnant women eat, try to eat more iron-rich foods, such as jujube, brown sugar, red beans, pig liver, etc., which can help pregnant women alleviate iron deficiency anemia [16] [17] [18] [19] [20]. When pregnant women usually eat, try to add some iron-rich foods, such as jujube, brown sugar, red beans, pig liver, and so on, which can help pregnant women to alleviate iron deficiency anemia [16] [17] [18] [19] [20]. The results of the study are reported below.

\section{Data and Methods}

\subsection{Object of Study}

15,656 study subjects were collected from all pregnant women, hospitalized gravidas, gynecological outpatient clinics and gynecological inpatients in our hospital from January 1, 2017, to October 28, 2020. Among them were 15,300 pregnant women with an average age of (28.04 \pm 6.14$)$ years old; 124 pregnant women were hospitalized with an average age of $(27.49 \pm 6.34)$ years old; 11 gynecological clinics with an average age of $(31.36 \pm 10.97)$ years old; 25 cases of gynecological hospitalization with an average age $(33.36 \pm 7.83)$. The age comparison of each group, the comparison between pregnant women during pregnancy and hospitalized women, $\mathrm{t}=0.9932, \mathrm{P}=0.1613$, the difference was not statistically significant; The age comparison of each group, the comparison between pregnant women during the examination and hospitalized pregnant women, $\mathrm{t}=0.9932, \mathrm{P}=0.1613$, the difference was not statistically significant; Comparison of pregnant women during the examination and pregnant women hospitalized in the different age groups, the difference was not statistically significant $(\mathrm{t}=0.9932, \mathrm{P}=0.1613)$.

The difference between pregnant women and gynecological hospitalization was statistically significant $(\mathrm{t}=2.8721, \mathrm{P}=0.0083)$; Comparing hospitalized parturient women with gynecological hospitalization, the difference was statistically significant $(\mathrm{t}=2.8865, \mathrm{P}=0.0074$; Comparing gynecological inpatient and gynecological outpatient, the difference was not statistically significant $(t=$ $0.5447, \mathrm{P}=0.2953$ ); Comparing prenatal examination of pregnant women and gynecology clinic, $\mathrm{t}=1.5128, \mathrm{P}=0.0717$, the difference was not statistically significant; Comparing hospitalized pregnant women and gynecological clinics, the difference was statistically significant $(t=1.7074, P=0.0496)$. Since most of the gynecological patients have already experienced the process of pregnancy and childbirth, they are older than pregnant women, so there is a certain difference in age between them, but it does not affect the monitoring results of disease screening indicators in this study. All study subjects were excluded from related underlying diseases, and the patients were required to sign an informed consent form before the study, and the study was conducted after approval by the ethics management committee of this hospital.

\subsection{Research Methods}

Select obstetric outpatients, inpatient maternal, gynecological inpatients and gy- 
necological outpatients as the research objects, and register the relevant information of the patients, such as specimen collection date, name, gender, age, department, test specimen number, etc., and all the subjects were collected venous blood on an empty stomach for blood routine and serum ferritin detection. Serum ferritin samples should be centrifuged in time after the blood is drawn and the test should be completed within 6 hours. If it cannot be tested in time, it must be stored at $-40^{\circ} \mathrm{C}$. After collecting a certain amount of specimens, they are uniformly reconstituted and tested; routine blood testing needs to be completed within 2 hours, and those that cannot be tested in time should be stored in a refrigerator at $2^{\circ} \mathrm{C}-8^{\circ} \mathrm{C}$, and testing should be completed within 24 hours. Then collect the test results, compare the differences of related indicators in each group, and put forward relevant conclusions.

\subsection{Reference Range and Clinical Significance of Various Indicators}

FER: male: about 15 - $200 \mathrm{ug} / \mathrm{L}$, female: about $12-150 \mathrm{ug} / \mathrm{L}$, iron overload: male $>336 \mu \mathrm{g} / \mathrm{L}$, female $>307 \mu \mathrm{g} / \mathrm{L}$, hemochromatosis ferritin $>1000 \mathrm{~g} / \mathrm{L}$; Serum ferritin is the most iron-rich protein in the body. The liver, spleen, red bone marrow and intestinal mucosa are the main places of iron storage, accounting for about $66 \%$ of the total iron in the body. The determination of serum ferritin is an important indicator to judge the iron storage in the body. Reduction: seen in iron deficiency anemia, blood loss, etc. (without complications); Increased: seen in malignant tumors, acute hepatitis, acute infection, chronic kidney disease, hemochromatosis, Gaucher disease, chronic inflammatory diseases, etc. [21] [22]; RBC: male: $(4.5-5.5) \times 10^{12} / \mathrm{L}$, female: $(3.5-5.4) \times 10^{12} / \mathrm{L}$, newborn: $(6$ - 7) $\times 10^{12} / \mathrm{L}$, increase: Physiological: newborns, high-altitude dwellers; Pathological: polycythemia vera, compensated polycythemia (such as congenital heart disease), Chronic lung disease, dehydration), reduce: various anemia, leukemia, various acute and chronic blood loss (such as: hemorrhoid bleeding, menorrhagia, digestive bleeding, massive bleeding after childbirth or surgery, hookworm disease); HGB: Male: 120 - 180 g/L, Female: 110 - 160 g/L, Newborn: 170 - 200 g/L, Increase: Physiological: High-altitude residents, Pathological: Polycythemia vera, compensated polycythemia, Reduction: various anemia, leukemia, acute and chronic blood loss, hemoglobin can better reflect the degree of anemia, such as iron deficiency anemia, the reduction of hemoglobin is more than the reduction of red blood cells; MCV: male: 80.0 - 94.0, female: 81.0 - 99.0, increase: acute hemolytic anemia, megaloblastic anemia; decrease: severe iron deficiency anemia, hereditary spherocytosis, sideroblastic anemia, thalassemia; $\mathrm{MCH}$ : 27 $31 \mathrm{pg}$, greater than normal: Megaloblastic anemia, hemolytic anemia, aplastic anemia; less than normal: iron deficiency anemia, sideroblastic anemia, thalassemia; MCHC: 320 - $360 \mathrm{~g} / \mathrm{l}$, greater than normal: megaloblastic anemia, hemolysis Sexual anemia, aplastic anemia; less than normal: iron deficiency anemia, sideroblastic anemia, thalassemia [23] [24] [25]. 


\subsection{Statistical Analysis}

The SPSS 24.0 software was used to perform statistical analysis on the obtained data. Age, blood routine indicators, and serum iron are all expressed in measurement units (X average $\pm \mathrm{s}$ ), and the results are compared using t-test; count data uses chi-square $\chi^{2}$ test and $\mathrm{P}<0.05$ is considered statistically significant.

\section{Results}

Among the 15656 study subjects collected, 15, 300 pregnant women were undergoing maternity examination, accounting for $97.73 \%$; 124 pregnant women were hospitalized, accounting for $0.79 \%$; 11 gynecological outpatient clinics, accounting for $0.007 \%$, and 25 were hospitalized in gynecology, accounting for $0.16 \%$; All subjects were tested for FER, RBC, HGB, MCV, $\mathrm{MCH}, \mathrm{MCHC}$ and other related indicators, as detailed in Table 1(a). Comparing the test index results of each group, comparing pregnant women with maternity checkups and hospitalized women, except for RBC, the differences in other indicators were statistically significant $(\mathrm{P}<0.05)$; There was no statistically significant difference in all indicators between the gynecological outpatient clinic and the gynecological hospitalization, the maternity check-up and the gynecological outpatient clinic, the inpatient maternal and the gynecological hospitalization and other groups $(\mathrm{P}>0.05)$. Compared with hospitalized puerperant and gynaecological hospitalization, except for MCV and $\mathrm{MCH}$, the differences in other indicators

Table 1. (a) Comparison of various indicators in each group of research objects; (b) Comparison of indicators in each group of research objects (continued table).

(a)

\begin{tabular}{|c|c|c|c|c|c|c|c|}
\hline Group & No. of cases & FER $(\mu \mathrm{g} / \mathrm{L})$ & HGB $(g / L)$ & $\mathrm{RBC}\left(\times 10^{12} / \mathrm{L}\right)$ & $\operatorname{MCV}(f l)$ & $\mathrm{MCH}(\mathrm{pg})$ & $\mathrm{MCHC}(\mathrm{g} / \mathrm{L})$ \\
\hline $\begin{array}{l}\text { Prenatal examination of } \\
\text { pregnant women }\end{array}$ & 15300 & $53.11 \pm 62.03$ & $111.82 \pm 11.77$ & $3.95 \pm 0.49$ & $86.93 \pm 9.31$ & $28.57 \pm 3.57$ & $327.30 \pm 14.32$ \\
\hline Hospitalized pregnant women ${ }^{2}$ & 124 & $32.35 \pm 45.22$ & $108.19 \pm 12.86$ & $3.92 \pm 0.46$ & $84.01 \pm 8.22$ & $27.81 \pm 3.36$ & $329.76 \pm 14.30$ \\
\hline Gynecology clinic ${ }^{3}$ & 11 & $145.9 \pm 264.35$ & $117.18 \pm 9.17$ & $4.16 \pm 0.61$ & $87.00 \pm 9.06$ & $28.59 \pm 3.62$ & $327.64 \pm 10.87$ \\
\hline Gynecological inpatients ${ }^{4}$ & 25 & $89.15 \pm 55.85$ & $119.68 \pm 15.92$ & $4.38 \pm 0.46$ & $81.92 \pm 10.29$ & $27.58 \pm 4.14$ & $335.84 \pm 14.92$ \\
\hline
\end{tabular}

(b)

\begin{tabular}{ccccccccccccccc}
\hline \multirow{2}{*}{$\begin{array}{c}\text { Comparison of } \\
\text { each group }\end{array}$} & \multicolumn{2}{c}{ FER $(\mu \mathrm{g} / \mathrm{L})$} & \multicolumn{2}{c}{$\mathrm{HGB}(\mathrm{g} / \mathrm{L})$} & \multicolumn{2}{c}{$\mathrm{RBC}\left(\times 10^{12} / \mathrm{L}\right)$} & \multicolumn{2}{c}{$\mathrm{MCV}(\mathrm{fl})$} & \multicolumn{2}{c}{$\mathrm{MCH}(\mathrm{pg})$} & \multicolumn{2}{c}{$\mathrm{MCHC}(\mathrm{g} / \mathrm{L})$} \\
\cline { 2 - 13 } & T value & P value & T value & P value & T value & P value & T value & P value & T value & P value & T value & P value \\
\hline 1,2 & 5.0737 & 0.0000 & 3.4178 & 0.0004 & 0.6793 & 0.2491 & 3.4816 & 0.0003 & 2.3621 & 0.0099 & 1.9053 & 0.0295 \\
$1,2,3$ & 1.1642 & 0.1357 & 1.5100 & 0.0810 & 1.4206 & 0.0929 & 0.0249 & 0.4903 & 0.0186 & 0.4928 & 0.0787 & 0.4694 \\
$1,2,3,4$ & 2.9031 & 0.0039 & 2.4675 & 0.0106 & 4.3846 & 0.0001 & 2.6880 & 0.0064 & 1.3850 & 0.0894 & 2.9792 & 0.0033 \\
2,3 & 1.4228 & 0.0926 & 2.2643 & 0.0207 & 1.6131 & 0.0675 & 1.1470 & 0.1379 & 0.7335 & 0.2393 & 0.4789 & 0.3200 \\
$2,3,4$ & 5.4984 & 0.0000 & 3.9090 & 0.0002 & 4.5613 & 0.0000 & 1.1095 & 0.1380 & 0.2998 & 0.3832 & 1.9255 & 0.0314 \\
$2,3,4$ & 0.7051 & 0.2484 & 0.4842 & 0.3158 & 1.1952 & 0.1253 & 1.4119 & 0.0863 & 0.6989 & 0.2461 & 1.6361 & 0.0569 \\
\hline
\end{tabular}

Note: For the convenience of statistics, the pregnant women in the obstetric examination are regarded as the ${ }^{1}$ group, the hospitalized pregnant women are the ${ }^{2}$ group, the gynecological outpatients are the ${ }^{3}$ group, and the gynecological inpatients are the ${ }^{4}$ group. 
were statistically significant $(\mathrm{P}<0.05)$; Comparing hospitalized puerperant and gynecological clinics, except for the difference of the HGB results which was statistically significant $(\mathrm{P}<0.05)$, the differences in other indicators were not statistically significant $(P>0.05)$. The specific results are shown in Table $1(b)$.

\section{Discussion}

After pregnancy, due to the growth and development of the fetus, women's demand for iron will increase. If the iron intake is insufficient, the serum ferritin value will decrease, leading to symptoms such as anemia, dizziness, and fatigue. During pregnancy, monitoring during pregnancy should be strengthened, and iron supplementation should be paid attention to. By monitoring the value of serum protein in the pregnant woman's blood, we can determine whether there is iron deficiency in the pregnant woman's body and too low or high will cause certain effects on the fetus and pregnant women. Ferritin has a normal reference range, that is, normal serum ferritin is $35-55 \mathrm{ug} / \mathrm{L}$. If the value is too low, it will easily cause iron deficiency anemia in pregnant women and affect the normal development of the fetus [26] [27] [28]. It can be supplemented by taking ferrous sulfate, iron dextran and other drugs, or supplemented by food and also can be supplemented by eating more iron-rich foods such as animal liver, red dates, fungus, spinach, and pig blood. The food should be diversified and not partial eclipse [29] [30] [31] [32]. Pregnant women with low ferritin: In the late pregnancy, with the rapid growth and development of the fetus in the uterus, the nutrients obtained from the mother will also greatly increase. Pregnant women in the late pregnancy have low ferritin, which is generally considered to be iron deficiency. If anemia is only low in ferritin content, don't worry too much. It can be improved by diet or iron supplementation. If there are other complications, such as inflammation, a certain amount of attention should be paid to avoid affecting fetal development and harm to the mother. High ferritin in pregnant women: high ferritin in pregnant women may lead to iron deficiency anemia, that is, high ferritin is one of the causes of anemia in pregnant women. In addition, high ferritin in pregnant women may also be caused by malignant tumors, such as lymphoma, leukemia and other diseases [33] [34] [35]. Therefore, pregnant women with high ferritin need to attract enough attention and actively treat different situations to avoid adverse effects on mothers and fetuses.

Routine blood examination during pregnancy is also a part of the pregnancy check-up. If you want to see if the pregnant woman is healthy, just check the blood routine checklist. As a pregnant woman in the new era, of course, she should pay special attention to her physical condition, and she should also know some common indicators. There are many indicators in blood routine, but we need to pay attention to several key points, namely WBC, RBC, HGB, MCV, $\mathrm{MCH}, \mathrm{MCHA}$ and PLT data. The main function of hemoglobin and red blood cells is to carry oxygen molecules through the blood circulation to provide oxygen to the baby. When the hemoglobin and red blood cells in the blood decrease, 
the expectant mother may be hypoxic and dehydrated; In severe cases, anemia may occur. Anemia in pregnant women is a common symptom, so at this time they are responsible for the health of two people. The blood volume of expectant mothers will increase than before pregnancy. In addition, at this time, the baby needs to take nutrients from the mother when growing up, so mild anemia can be adjusted with diet, but severe anemia must be treated in time, which can easily cause premature delivery or congenital insufficiency of the fetus. This study mainly understands blood routine items related to serum ferritin, so it focuses on observing the changes of RBC, $\mathrm{HGB}, \mathrm{MCV}, \mathrm{MCH}$, and $\mathrm{MCHC}$.

The results of this study show that the comparison between maternity check-ups and hospitalized pregnant women, except for RBC, the differences in other indicators are statistically significant $(\mathrm{P}<0.05)$; this indicates that the serum ferritin and blood routine-related indicators of pregnant women are at a relatively stable level during pregnancy which is the result of timely treatment of problems found by pregnancy screening during pregnancy. However, due to the increase of nutrients needed to nourish the fetus in the later stage of pregnancy, the hospitalized parturient increased the burden of the mother, resulting in excessive consumption of ferritin, increased blood volume and prone to iron deficiency anemia, so all the indexes were at a lower level. There was no statistically significant difference in all indicators between the gynecological outpatient clinic and the gynecological hospitalization, the maternity check-up and the gynecological outpatient clinic, the inpatient maternal and the gynecological hospitalization $(\mathrm{P}>0.05)$. Comparing hospitalized parturients with gynecological hospitalizations, except for $\mathrm{MCV}$ and $\mathrm{MCH}$, the differences in other indicators are statistically significant $(\mathrm{P}<0.05)$. This indicates that the serum ferritin and blood routine related indicators of hospitalized women are lower than those of general gynecological patients. The reason is still related to the increase in nutrients needed by women to nourish the fetus in the later stages of pregnancy, which increases the burden on the mother, resulting in excessive ferritin consumption and increased blood volume. Comparing hospitalized parturients with gynecological clinics, except for the statistically significant difference in HGB results ( $\mathrm{P}$ $<0.05)$, the differences in other indicators were not statistically significant $(\mathrm{P}>$ $0.05)$, which indicates that the increased nutrition of the fetus in the hospitalized parturients in the later period led to a decrease in HGB which was compared with the difference caused by low hemoglobin in patients with normal gynecological diseases. When iron-deficiency anemia occurs in pregnant women, the effect of treatment can be achieved through diet control. At present, it is believed that diet therapy is the safest and also very effective. Pregnant women with mild anemia can eat more animal livers which are rich in iron and the vitamin content is also very good. Light cooking can allow the body to absorb nutrients and stick to the methods for a while then make another routine pregnancy test, you can see the increase in hemoglobin and red blood cell index. Simple low ferritin or anemia caused by high ferritin can be improved by iron supplementation or 
blood transfusion. In addition, diet regulation can be carried out, and more iron-containing foods can be eaten, or foods conducive to iron absorption.

The treatment of the above methods will generally receive good results. Low ferritin and inflammation, or high blood protein caused by malignant tumors, go to a regular hospital for examination, and follow the doctor's instructions for active treatment under the guidance of a doctor. A certain amount of attention should be paid, but not too worried about anxiety, face up to the problems that arise during pregnancy, and actively solve them [36] [37] [38].

\section{Conclusion}

During the maternity examination of pregnant women, ferritin is consumed too much, blood volume increases, and blood routine-related items are reduced during the maternity examination. The consumption is greater for a period of time after delivery, and recovery requires a certain process, which is relatively low. After the newborn is born, the ferritin recovered slowly and the concentration of ferritin increased. This process is a physiological process and does not involve related diseases, but from this result, we are also required to strengthen nutrition for pregnant women in order to give birth to healthier and healthy babies. The ferritin test of gynecological patients is mainly a screening of related diseases. The results of this time did not screen out serious diseases related to ferritin changes.

\section{Limitations of Research}

This research mainly studies the region serum ferritin in combination with related parameters of routine blood test in the application of obstetrics and gynecology disease screening, although the sample size is more, but is given priority to with prenatal women, other maternal hospitalization, outpatient service of department of gynaecology, hospital of department of gynaecology such as sample size is less, do not represent all of the patients, so there are some limitations. The solution can be to increase the sample size of inpatient puerpera, outpatient department of gynecology and inpatient department of gynecology, so that the data statistics can be balanced to reflect the correlation between the groups.

\section{Acknowledgements}

The project was approved with the strong support of the functional departments of the hospital and the help of colleagues. In the process of implementation, the project also received the strong help and support of colleagues in the department, patients and patients' families. Here, I would like to express my heartfelt thanks to their support and help! I wish them good health, smooth work and all the best!

\section{Fund Project}

Scientific Research and Technology Development Program of Yulin City, Gua- 
ngxi Province (Yulin Science \& Technology 20171624).

\section{Conflicts of Interest}

The authors declare no conflicts of interest regarding the publication of this paper.

\section{References}

[1] Lu, H.M. (2020) Application of Red Blood Cell Parameters Combined with Serum Ferritin in Diagnosis of Iron Deficiency Anemia. China Health Nutrition, 30, 3.

[2] Liao, G.J. (2020) Application of Hemoglobin Electrophoresis, Serum Ferritin and Blood Routine Examination in Differential Diagnosis of Thalassemia and Iron Deficiency Anemia. Dietary Care, 7, 225-226.

[3] Wang, S.W. (2020) Study on the Value of Combined Detection of Serum Ferritin, Folic Acid and Vitamin B12 on Megaloblastic Anemia. China Health Nutrition, 30, 130.

[4] Chen, Z.Y. (2020) Relationship between Dietary Inhibitory Factor-1 and Serum Ferritin and Gestational Diabetes Mellitus during Early and Middle Pregnancy. Journal of Hebei North University (Natural Science Edition), 36, 26-2830.

[5] Qian, L.L. and Hong, X.Z. (2020) Serum Ferritin Level, Iron Deficiency Anemia in Pregnant Women and Its Effect in Late Pregnancy Outcome in Yueqing Area. China Maternal and Child Health Care, 35, 995-998.

[6] Zhang, J.F. (2020) The Value of Dynamic Detection of Serum Ferritin, Vitamin B12/ Folic Acid Ratio in the Evaluation of Efficacy of Iron Deficiency Anemia Patients. Henan Medical Research, 29, 5699-5701.

[7] Li, X.X. (2020) The Influence of Maternal Serum Ferritin, Folic Acid and Vitamin B12 Levels on Maternal Anemia during Pregnancy. Channel Pharmacy, 32, 134-136.

[8] Xiong, J.X. (2020) Diagnostic Value of Prealbumin Combined with Serum Iron and Ferritin in Iron Deficiency Anemia. A Systems Medicine, 5, 50-52.

[9] Tang, J. (2020) The Clinical Significance of Serum Ferritin, Folic Acid and Vitamin B12 Expression in Different Pregnancy Stages of Pregnancy. Medical Aesthetic Beauty, 29, 81-82.

[10] Wang, Y.M., Chen, Y. and Li, Q. (2020) Correlation Study of Serum Iron, Ferritin and Transferrin with Gestational Diabetes Mellitus. Laboratory Medicine and Clinical Science, 17, 1030-1032.

[11] Liu, K.L., Chen, Y.P., Mo, X.L., et al. (2020) Effects of Thyroid Hormone Reference Value and Serum Ferritin and 25-Hydroxyvitamin D Levels on Thyroid Function during Early Pregnancy in Nanning Area. Journal of Guangxi Medical University, 37, 911-916.

[12] Li, S.J., Zhu, H.Q. and Liao, J.L. (2020) Analysis of the Correlation between Serum Estradiol and Ferritin Levels and Postmenopausal Bone Mineral Density in Women. China Practical Medicine, 15, 26-28.

[13] Yang, L.Q. (2019) Study on the Results of Serum Ferritin Detection of Pregnant Women in Lingshan Area of Guangxi. Diet \& Health Care, 6, 294.

[14] Lin, D.J. (2020) The Effect of Polysaccharide Iron Complex on the Curative Effect and Serum Ferritin Level in Patients with Iron Deficiency Anemia. Health Vision, No. $8,21$.

[15] Liang, X.H. (2020) The Correlation between Serum Ferritin Levels in Pregnant 
Women with Gestational Diabetes and Insulin Resistance and Maternal and Infant Outcomes. Clinical Medical Engineering, 27, 1621-1622.

[16] Li, Y.C. (2020) The Value of Combined Detection of Serum VitB12 and Ferritin in the Diagnosis and Differential Diagnosis of Anemia. Oriental Medicinal Diet, No. 7, 82 .

[17] Cao, D.H. (2019) The Association Study of Maternal Serum Ferritin, CRP, C3 and C4 Levels and the Risk of Singleton Low Birth Weight Infants. Guangxi Medical University, Guangxi.

[18] Liu, W.X. (2019) To Study the Efficacy of Polysaccharide Iron Complex in the Treatment of Iron Deficiency Anemia and Its Effect on Serum Transferrin Receptor and Serum Ferritin. Contemporary Medicine, 25, 57-59.

[19] Chen, K.Y., Tang, B.J., Jiang, H.Z., et al. (2019) Significance of Combined Detection of Bone Marrow Iron Staining, Red Blood Cell Indicators, and Serum Ferritin in the Diagnosis of Iron Deficiency Anemia. Journal of Clinical Rational Use, 12, 158-159.

[20] Yao, Y.C. (2019) The Changes and Clinical Significance of Serum Ferritin, Folic Acid and Vitamin B12 Levels in Pregnant Women during Different Pregnancy Periods. Family Medicine, No. 9, 116-117.

[21] Chen, C.N., Huang, P.B. and Wu, H.Y. (2019) The Relationship between Serum iron, Ferritin, Soluble Transferrin Receptor Levels and Anemia during Pregnancy. Chinese Sexual Sciences, 28, 49-52.

[22] Du, J. (2019) Analysis of the Relationship between Serum Folate, Vitamin B12, Ferritin Levels and Pregnancy Complications in Pregnant Women. Jiayou Yunbao, 1, 36-37.

[23] Fang, C. and Liu, P. (2019) The Relationship between Serum Ferritin Levels and Changes in Early and Middle Pregnancy and Gestational Diabetes. The PLA Journal of Preventive Medicine, 37, 67-68.

[24] Yang, J.M. and Ou, L.J. (2019) The Effect of MCV, MCH, and Serum Ferritin Testing in Thalassemia Screening. Oriental Medicinal Food, No. 15, 117-118.

[25] Xiao, Y.H. and Yi, S. (2019) Analysis of the Characteristics of Serum Ferritin Levels and Anemia Prevalence of Pregnant Women in Different Trimesters. China Maternal and Child Health Care, 34, 4886-4888.

[26] Ning, N. (2019) Study on the Correlation between Serum Iron, Serum Ferritin and Hemoglobin in Women in the Middle and Late Stages of Pregnancy. Diet and Health Care, 6, 23.

[27] Huang, Y. and Wang, Y. (2019) The Relationship between Serum Ferritin Levels and Changes in Early and Middle Pregnancy and Gestational Diabetes. Psychological Monthly, 14, 140-142.

[28] Wang, S.W. (2020) Clinical Analysis of Changes in Serum Ferritin, Folic Acid and Vitamin B12 Levels in Pregnant Women during Different Gestational Periods. Friends of Health, No. 4, 65.

[29] Wang, Y. (2019) The Clinical Value of Early Pregnancy Fasting Blood Glucose, Triglycerides, and Serum Ferritin in Predicting the Risk of Gestational Diabete. Soochow University, Jiangsu.

[30] Hou, F. (2019) Study on the Correlation between Serum 25-Hydroxyvitamin D, Ferritin and Glucose and Lipid Metabolism in Pregnant Women. Zunyi Medical University, Zunyi.

[31] Shi, N. (2019) Study on the Correlation between Serum Iron, Ferritin and Hypothyroidism during Pregnancy. Zhengzhou University, Zhenzhou. 
[32] Gu, D.Q. (2020) Clinical Significance of Serum Ferritin Detection in Patients with Anemia during Perioperative Period of Gynecology. Hebei University of Technology, Shijiazhuang.

[33] Wang, Z.H. (2019) Research Progress on the Relationship between Serum Ferritin and Iron Deficiency Anemia in Pregnant Women. China Health Care and Nutrition, 29, 20.

[34] Luo, X.H., Shi, N., Guo, X.P., et al. (2019) Correlative Research on Serum Iron and Serum Ferritin Levels in Pregnant Women. Chinese Journal of Maternal and Child Health, 10, 35-38.

[35] Li, M., Zhao, Y., Ma, D. and Dong, B.C. (2020) Serum Ferritin and Homologous Antagonists Expression and Clinical Significance in Postmenopausal Women with Osteoporosis. Shanxi Medical Journal, 49, 1266-1269.

[36] Liu, Y.L. (2020) Effectiveness Analysis of Combined Detection of Serum VitB12 and Ferritin for Clinical Diagnosis and Identification of Anemia. Gansu Science and Technology, 36, 128-130.

[37] Gu, H., Chen, Y.J., Xiong, A.Q., et al. (2019) The Clinical Significance of Average Red Blood Cell Volume, Average Red Blood Cell Hemoglobin Content, and Serum Ferritin Detection for Iron Deficiency Anemia in Pregnant Women. Electronic Journal of Practical Gynecology and Endocrinology, 6, 108, 113.

[38] Guan, X.H. (2020) The Diagnostic Value of Blood Routine Examination and Serum Ferritin Examination in the Infection of Iron Deficiency Anemia. China Contemporary Medicine, 27, 204-206. 